\title{
Finite Fourier Decomposition of Signals Using Generalized Difference Operator
}

\author{
G. B. A. Xavier, B. Govindan, S. J. Borg, M. Meganathan
}

\begin{abstract}
In this paper, we introduce discrete inner product of two functions, discrete orthogonal and orthonormal system of functions and develop finite Fourier series for polynomial factorial, polynomial, exponential, rational and logarithm functions using the inverse of generalized difference operator
\end{abstract}

Keywords: Discrete inner product, Discrete orthonormal system, Finite Fourier series and Generalized difference operator.

\section{Introduction}

In Fourier analysis, a signal is decomposed into its constituent sinusoids. In the reverse by operating the inverse Fourier transform, the signal can be synthesized by adding up its constituent frequencies. Many signals that we encounter in daily life such as speech, automobile noise, chirps of birds, music etc. have a periodic or quasi-periodic structure, and that the cochlea in the human hearing system performs a kind of harmonic analysis of the input audio signals in biological and physical systems [11]. The Fourier series decomposes the given input signals into a sum of sinusoids. By removing the high frequency terms(noise) of Fourier series and then adding the remaining terms can yield better signals [4].

Finite Fourier series is a powerful tool for attacking many problems in the theory of numbers. It is related to certain types of exponential and trigonometric sums. It may therefore be expanded into a finite Fourier series of the form

$f\left(\alpha^{\mu}\right)=\sum_{j=0}^{m-1} g(j) \alpha^{\mu j}(\mu=0,1, \cdots, m-1)$. The orthogonality relation

$\sum_{j=0}^{m-1} \alpha^{a j} \alpha^{-b j}=\left\{\begin{array}{ll}m & (a \equiv b(\bmod m)), \\ 0 & (a \neq b(\bmod m)),\end{array} \quad\right.$ enables us to determine the finite Fourier coef-

Manuscript received March 21, 2016; accepted August 29, 2016.

G. B. A. Xavier, B. Govindan, S.J. Borg, M. Meganathan are with the Department of Mathematics,Sacred Heart College, Tirupattur - 635601, Vellore District, Tamil Nadu, S. India 
ficients $g(k)$ explicitly by means of the formula $g(k)=\frac{1}{m} \sum_{\mu=0}^{m-1} f\left(\alpha^{\mu}\right) \alpha^{-\mu k}$ [2]. If we are given $k$ distinct complex numbers $z_{0}, z_{1}, \cdots, z_{k-1}$, then there is one and only one polynomial $P(x)=\zeta_{0}+\zeta_{1} x+\cdots+\zeta_{k-1} x^{k-1}$ satisfying the equations $P\left(\omega_{v}\right)=z_{v}(v=0,1, \cdots, k-1)$ [10].

A finite Fourier series: $\eta(t)=A_{0}+\sum_{q=1}^{N / 2} A_{q} \cos \left(q \sigma_{1} t\right)+\sum_{q=1}^{N / 2-1} B_{q} \sin \left(q \sigma_{1} t\right)$, where $\eta=$ sea surface elevation $(m), t=$ time $(s), A_{0}=$ recond mean $(m), N=$ total number of sampling points, $A_{q}$ and $B_{q}=$ Fourier coefficients $(m), q=$ harmonic component index (in the frequency domain) and $\sigma_{1}=$ fundamental radian frequency, is used in [6]. The sum of $N$ sine waves defined over the time interval, $0 \leq t \leq T: y=\sum_{n=1}^{N} a_{n} \cos \left(\omega_{n} t+\phi_{n}\right), 0 \leq t_{n} \leq T$, $a_{n} \geq 0,0 \leq \phi_{n}<2 \pi$, where $a_{n}$ is amplitude and $t$ is time, is also a finite Fourier series[3]. In [7], the authors describe an efficient formulation, based on a discrete Fourier series expansion, of the analysis of axi-symmetric solids subjected to non-symmetric loading. They have discussed the Fourier series approach, the discrete Fourier series representation problems such as the presence of Gibb's phenomenon and the lack of conformity of elements. Here we arrive a new type of finite Fourier series for functions (signals) by defining discrete orthonormal family of functions using inverse generalized difference operator $\Delta_{\ell}^{-1}$. This finite Fourier series becomes Fourier series as $\ell$ tends to zero. Suitable examples verified by MATLAB are inserted to illustrate the findings.

\section{Preliminaries}

The primitive $N^{\text {th }}$ roots of unity $\left(z^{N}=1\right.$ but $\left.z^{r} \neq 1 ; 0<r<N\right)$

$$
z_{n}=e^{i(2 \pi / N) n}, n=1,2,3, \ldots, N-1,
$$

where $n$ and $N$ are co-prime, satisfies the geometric series expressed as

$$
\sum_{k=0}^{N-1} z_{n}^{k}=\left.\Delta^{-1} z_{n}^{k}\right|_{k=0} ^{N}=\frac{z_{n}^{N}-1}{z_{n}-1}=\left\{\begin{array}{lll}
1 & \text { if } & N=1 \\
0 & \text { if } & N>1
\end{array}\right.
$$

From (1) and (2), the complex discrete-time sequence $e_{r}(k)$ is defined as

$$
e_{n}(k)=\left(z_{n}\right)^{k}=e^{i(2 \pi / N) n k} ; n, k=0,1,2, \ldots, N-1 .
$$

For the positive integers $n, r$ and $N$, the $e_{n}(k)$ defined in (3) satisfies the identity

$$
\sum_{k=0}^{N-1} e_{n}(k)=\left.\Delta^{-1} e_{n}(k)\right|_{k=0} ^{N}=\left.\Delta^{-1} e^{i(2 \pi n / N) k}\right|_{k=0} ^{N}=\left\{\begin{array}{lll}
N & \text { if } & n=r N \\
0 & \text { if } & n \neq r N
\end{array}\right.
$$


This mathematical property is utilized with the factorization into two orthogonal exponential functions, $\left\{e_{n}(k)\right\}$ satisfying

$$
\left.\Delta^{-1} e_{n}(k) e_{m}^{*}(k)\right|_{k=0} ^{N}=\left.\Delta^{-1} e^{i\left(\frac{2 \pi(n-m) k}{N}\right)}\right|_{k=0} ^{N}=\left\{\begin{array}{lcc}
N & \text { if } & n-m=r N \\
0 & \text { if } & n-m \neq r N
\end{array}\right.
$$

where $m, n$ and $r$ are integers, and the notation $(*)$ represents the complex conjugate. The equation (5) induces us to define a generalized discrete orthonormal system and a finite Fourier series by replacing $\Delta$ by $\Delta_{\ell}$ and $e_{n}(k)$ by $u_{n}(k)$. Nonexistence of solutions of certain type of second order generalized $\alpha$-difference equation with the operator $\Delta_{\alpha(\ell)}$ has been discussed in [9]. When $\alpha=1$ the operator $\Delta_{\alpha(\ell)}$ becomes the generalized difference operator $\Delta \ell$.

Definition 1 [8] Let $u(k), k \in[0, \infty)$, be a real or complex valued function and $\ell>0$ be fixed. Then, the generalized difference operator $\Delta_{\ell}$ on $u(k)$ is defined as

$$
\Delta_{\ell} u(k)=u(k+\ell)-u(k),
$$

and its inverse is defined as if there is a function $v(k)$ such that

$$
\Delta_{\ell} v(k)=u(k), \text { then } v(k)=\Delta_{\ell}^{-1} u(k)+c_{j}, \text { for } k \in\{j+r \ell\}_{r=0}^{\infty},
$$

where $c_{j}$ is constant, $j=k-[k / \ell] \ell$ and $[k / \ell]$ is the integer part of $k / \ell$.

Lemma 1 [8] Let $s_{r}^{m}$ and $S_{r}^{m}$ are the Stirling numbers of first and second kinds, $k_{\ell}^{(0)}=1$, $k_{\ell}^{(1)}=k$ and $k_{\ell}^{(m)}=k(k-\ell)(k-2 \ell) \cdots(k-(m-1) \ell)$. Then we have

$$
k_{\ell}^{(m)}=\sum_{r=1}^{m} s_{r}^{m} \ell^{m-r} k^{r}, k^{m}=\sum_{r=1}^{m} S_{r}^{m} \ell^{m-r} k_{\ell}^{(r)}, \Delta_{\ell} k_{\ell}^{(m)}=(m \ell) k_{\ell}^{(m-1)}
$$

and

$$
\Delta_{\ell}^{-1} k_{\ell}^{(m)}=\frac{k_{\ell}^{(m+1)}}{\ell(m+1)}, \Delta_{\ell}^{-1} k^{m}=\sum_{r=1}^{m} \frac{S_{r}^{m} \ell^{m-r} k_{\ell}^{(r)}}{(r+1) \ell} .
$$

Lemma 2 [5] Let $p$ be real, $\ell>0, k \in(\ell, \infty)$ and $p \ell \neq m 2 \pi$. Then, we have

$$
\begin{gathered}
\Delta_{\ell}^{-1} \sin p k=\frac{\sin p(k-\ell)-\sin p k}{2(1-\cos p \ell)}+c_{j}, \\
\Delta_{\ell}^{-1} \cos p k=\frac{\cos p(k-\ell)-\cos p k}{2(1-\cos p \ell)}+c_{j}
\end{gathered}
$$

and

$$
\Delta_{\ell}^{-1}(u(k) w(k))=v(k) \Delta_{\ell}^{-1} w(k)-\Delta_{\ell}^{-1}\left(\Delta_{\ell}^{-1} w(k+\ell) \Delta_{\ell} u(k)\right) .
$$


Remark 1 From (10) and (11), we have

$$
\left.\Delta_{\ell}^{-1} \sin p k\right|_{0} ^{2 \pi}=0=\left.\Delta_{\ell}^{-1} \cos p k\right|_{0} ^{2 \pi} .
$$

Lemma 3 [8] If $u(k)$ is a bounded function on $[a, b]$ and $\ell=\frac{b-a}{M}$, then we have

$$
\left.\Delta_{\ell}^{-1} u(k)\right|_{a} ^{b}=\sum_{r=1}^{M} u(b-r \ell)=\sum_{r=0}^{M-1} u(a+r \ell)
$$

In general, when $k \in(\ell, \infty)$, we express

$$
\left.\Delta_{\ell}^{-1} u(k)\right|_{j} ^{k}=\sum_{r=1}^{\left[\frac{k}{\ell}\right]} u(k-r \ell)=\sum_{r=0}^{\left[\frac{k}{\ell}\right]-1} u(j+r \ell), j=k-[k / \ell] \ell .
$$

\section{Discrete Orthogonal System and Finite Fourier Series}

Since a finite Fourier series is described by a family of discrete orthonormal functions, we introduce a orthogonal and further a orthonormal system of functions by defining the discrete inner product.

Definition 2 Let $u(k)$ and $v(k)$ be bounded functions defined on $[a, b]$ and $\ell=\frac{b-a}{M}$. The discrete inner product of $u$ and $v$ with respect to $\ell$ is defined as

$$
(u, v)_{\ell}=\left.\ell \Delta_{\ell}^{-1} u(k) v^{*}(k)\right|_{a} ^{b}=\ell \sum_{r=0}^{M-1} u(a+r \ell) v^{*}(a+r \ell)
$$

and the discrete $\ell-$ norm of $u$, denoted by $\|u\|_{(\ell)}$ is defined as

$$
\|u\|_{(\ell)}=(u, u)_{\ell}^{1 / 2}=\left\{\left.\ell \Delta_{\ell}^{-1}|u(k)|^{2}\right|_{a} ^{b}\right\}^{1 / 2}=\left\{\ell \sum_{r=0}^{M-1}|u(a+r \ell)|^{2}\right\}^{1 / 2} .
$$

Definition 3 Let $I=[a, b], \ell=\frac{b-a}{M}$ and $S_{\ell}=\left\{\phi_{0}, \phi_{1}, \phi_{2}, \ldots \phi_{M}\right\}$ be a collection of bounded complex valued functions defined on I. If $\left(\phi_{n}, \phi_{m}\right)_{\ell}=0$ whenever $m \neq n$, the collection $S_{\ell}$ is said to be a discrete orthogonal system, if in addition $\left\|\phi_{n}\right\|_{\ell}=1$ for each $n$, then $S_{\ell}$ is said to be discrete orthonormal. 
Definition 4 Let $S_{\ell}=\left\{\phi_{0}, \phi_{1}, \phi_{2}, \ldots \phi_{M}\right\}$ be an orthonormal on $I=[a, b], \ell=\frac{b-a}{M}, u(k)$ is a bounded function on $I$ and $c_{n}=\left(u, \phi_{n}\right)_{\ell}$, (say finite Fourier coefficients). Then the finite Fourier series of $u(k)$ related to $S_{\ell}$ is defined as

$$
u(k)=\sum_{n=0}^{M} c_{n} \phi_{n}(k), k \in\{a+r \ell\}_{r=0}^{M-1} .
$$

Example 1 Let $I=[a, a+2 \pi], \ell=\frac{\pi}{N}($ here $M=2 N)$ and

$$
\phi_{0}(k)=\frac{1}{\sqrt{2 \pi}}, \phi_{2 n-1}(k)=\frac{\sin n k}{\sqrt{\pi}}, \phi_{2 n}(k)=\frac{\cos n k}{\sqrt{\pi}}, n=1,2, \cdots, N .
$$

By (13) and $\left.\Delta_{\ell}^{-1} k_{\ell}^{(0)}\right|_{a} ^{a+2 \pi}=\frac{2 \pi}{\ell}$, we find that $S_{\ell}=\left\{\phi_{0}, \phi_{1}, \phi_{2}, \ldots \phi_{2 N}\right\}$ is a system of discrete orthonormal functions on $I$.

From (18), the finite Fourier series related to (19) is given by

$$
u(k)=\frac{a_{0}}{2}+\sum_{n=1}^{N-1}\left(a_{n} \cos n k+b_{n} \sin n k\right)+\frac{a_{N}}{2} \cos N k, k \in\{a+r \ell\}_{r=0}^{2 N-1},
$$

where $a_{n}=\left.\frac{\ell}{\pi} \Delta_{\ell}^{-1}(u(k) \cos n k)\right|_{a} ^{a+2 \pi}, b_{n}=\left.\frac{\ell}{\pi} \Delta_{\ell}^{-1} u(k) \sin n k\right|_{a} ^{a+2 \pi}$ are got by (14).

Theorem 3.1 Let $u(k)=\sum_{n=0}^{M} c_{n} \phi_{n}(k), k \in\{a+r \ell\}_{r=0}^{M-1}$ be the finite Fourier series of $u(k)$ relative to a discrete orthonormal set $S_{\ell}$. Then we have

$$
\sum_{n=0}^{M}\left|c_{n}\right|^{2}=\|u\|_{(\ell)}^{2} \quad \text { (Discrete Parseval's Formula) }
$$

Proof Since $S_{\ell}$ is orthonormal and $\Delta_{\ell}^{-1}$ is linear, (21) follows from (16), (17), (18) and the Definition 3.

Theorem 3.2 Let $k \in(-\infty, \infty)$ and $\ell>0$. If $n \ell \neq 2 m \pi$, then we have

$$
\Delta_{\ell}^{-1} k_{\ell}^{(m)} \cos n k=\sum_{t=0}^{m} \sum_{r=0}^{t+1}\left(\begin{array}{c}
t+1 \\
r
\end{array}\right) \frac{(m)_{1}^{(t)} \ell^{t} k_{\ell}^{(m-t)} \cos n(k-\ell+r \ell)}{(-1)^{r-1}(2(\cos n \ell-1))^{t+1}}
$$

and

$$
\Delta_{\ell}^{-1} k_{\ell}^{(m)} \sin n k=\sum_{t=0}^{m} \sum_{r=0}^{t+1}\left(\begin{array}{c}
t+1 \\
r
\end{array}\right) \frac{(m)_{1}^{(t)} \ell^{t} k_{\ell}^{(m-t)} \sin n(k-\ell+r \ell)}{(-1)^{r-1}(2(\cos n \ell-1))^{t+1}} .
$$


Proof Taking $u(k)=k_{\ell}^{(1)}, w(k)=\cos n k$ in (12) and using (9) and (11), we get $\Delta_{\ell}^{-1}\left(k_{\ell}^{(1)} \cos n k\right)=k_{\ell}^{(1)}\left(\frac{\cos n(k-\ell)-\cos n k}{2(1-\cos n \ell)}\right)-\Delta_{\ell}^{-1}\left(\frac{\cos n k-\cos n(k+\ell)}{2(1-\cos n \ell)} \ell\right)$.

Since $\Delta_{\ell}^{-1}$ is linear, applying (11) for both $\cos n k$ and $\cos n(k+\ell)$, we get $\Delta_{\ell}^{-1}\left(k_{\ell}^{(1)} \cos n k\right)=k_{\ell}^{(1)}\left(\frac{\cos n(k-\ell)-\cos n k}{2(1-\cos n \ell)}\right)$

$$
-\frac{\ell(\cos n(k-\ell)-2 \cos n k+\cos n(k+\ell))}{(2(1-\cos n \ell))^{2}} .
$$

Taking $u(k)=k_{\ell}^{(2)}, w(k)=\cos n k$ in (12), and using (9), (11) and (24), we get

$$
\begin{aligned}
\Delta_{\ell}^{-1}\left(k_{\ell}^{(2)} \cos n k\right)= & \frac{k_{\ell}^{(2)}(\cos n(k-\ell)-\cos n k)}{2(1-\cos n \ell)} \\
& -\frac{2 \ell k_{\ell}^{(1)}(\cos n(k-\ell)-2 \cos n k+\cos n(k+\ell))}{(2(1-\cos n \ell))^{2}} \\
+ & \frac{(1 \ell)(2 \ell)(\cos n(k-\ell)-3 \cos n k+3 \cos n(k+\ell)-\cos n(k+2 \ell))}{(2(1-\cos n \ell))^{3}}
\end{aligned}
$$

which can be expressed as

$\Delta_{\ell}^{-1} k_{\ell}^{(2)} \cos n k=\sum_{t=0}^{2} \sum_{r=0}^{t+1}\left(\begin{array}{c}t+1 \\ r\end{array}\right) \frac{(2)_{1}^{(t)} \ell^{t} k_{\ell}^{(2-t)} \cos n(k-\ell+r \ell)}{(-1)^{r-1}(2(\cos n \ell-1))^{t+1}}$.

Continuing the above process, we get the relation (22).

Now, (23) follows by replacing $\cos n k$ by $\sin n k$ in (22).

Corollary 1 When $I=[0,2 \pi], \ell=\frac{\pi}{N}, k \in\{r \ell\}_{0}^{2 N-1}$, the finite Fourier coefficients $a_{n}$ and $b_{n}$ for the polynomial factorial $k_{\ell}^{(m)}$ are given by

$$
\begin{gathered}
a_{0}=\left.\frac{\ell}{\pi} \Delta_{\ell}^{-1} k_{\ell}^{(m)}\right|_{0} ^{2 \pi}=\frac{(2 \pi)_{\ell}^{(m+1)}}{\pi(m+1)}, \\
a_{n}=\left.\frac{\ell}{\pi} \Delta_{\ell}^{-1} k_{\ell}^{(m)} \cos n k\right|_{0} ^{2 \pi}=\sum_{t=0}^{m-1} \sum_{r=0}^{t+1}\left(\begin{array}{c}
t+1 \\
r
\end{array}\right) \frac{(m)_{1}^{(t)} \ell^{t}(2 \pi)_{\ell}^{(m-t)} \cos n(r-1) \ell}{N(-1)^{r-1}(2(\cos n \ell-1))^{t+1}}
\end{gathered}
$$

and

$$
b_{n}=\left.\frac{\ell}{\pi} \Delta_{\ell}^{-1} k_{\ell}^{(m)} \sin n k\right|_{0} ^{2 \pi}=\sum_{t=0}^{m-1} \sum_{r=0}^{t+1}\left(\begin{array}{c}
t+1 \\
r
\end{array}\right) \frac{(m)_{1}^{(t)} \ell^{t}(2 \pi)_{\ell}^{(m-t)} \sin n(r-1) \ell}{N(-1)^{r-1}(2(\cos n \ell-1))^{t+1}} .
$$


Proof The proof follows by applying the limit 0 to $2 \pi$ in (22) and (23), and then multiplying by $\ell / \pi$.

Example 2 From (20), and using (26), (27) and (28) for $a_{0}, a_{n}$ and $b_{n}$ respectively, we get the finite Fourier series for the polynomial factorial $k_{\ell}^{(m)}$ as

$$
k_{\ell}^{(m)}=\frac{a_{0}}{2}+\sum_{n=1}^{N-1}\left(a_{n} \cos n k+b_{n} \sin n k\right)+\frac{a_{N}}{2} \cos N k, k \in\{r \ell\}_{r=0}^{2 N-1} .
$$

In particular, when $N=20, \ell=\frac{\pi}{20}$ and $m=15$, (29) becomes,

$$
(k)_{\pi / 20}^{(15)}=\frac{(2 \pi)_{\pi / 20}^{16}}{32 \pi}+\sum_{n=1}^{19}\left(a_{n} \cos n k+b_{n} \sin n k\right)+\frac{a_{20}}{2} \cos 20 k, k \in\left\{\frac{r \pi}{20}\right\}_{r=0}^{39} .
$$

Theorem 3.3 Let $k \in(-\infty, \infty), \ell>0$ and $n \ell \neq m 2 \pi$, then we have

$$
\Delta_{\ell}^{-1} k^{p} \cos n k=\sum_{m=1}^{p} \sum_{t=0}^{m} \sum_{r=0}^{t+1}\left(\begin{array}{c}
t+1 \\
r
\end{array}\right) \frac{S_{m}^{p}(m)_{1}^{(t)} k_{\ell}^{(m-t)} \cos n(k-\ell+r \ell)}{(-1)^{r-1} \ell^{m-t-p}(2(\cos n \ell-1))^{t+1}}
$$

and

$$
\Delta_{\ell}^{-1} k^{p} \sin n k=\sum_{m=1}^{p} \sum_{t=0}^{m} \sum_{r=0}^{t+1}\left(\begin{array}{c}
t+1 \\
r
\end{array}\right) \frac{S_{m}^{p}(m)_{1}^{(t)} k_{\ell}^{(m-t)} \sin n(k-\ell+r \ell)}{(-1)^{r-1} \ell^{m-t-p}(2(\cos n \ell-1))^{t+1}} .
$$

Proof The proof follows by second term of (8) and applying (22).

Corollary 2 When $I=[0,2 \pi], \ell=\frac{\pi}{N}$, the finite Fourier coefficients $a_{n}$ and $b_{n}$ for $n=$ $0,1,2, \cdots, N$ for polynomial $k^{p}$ are given by

$$
a_{n}=\left.\frac{\ell}{\pi} \Delta_{\ell}^{-1} k^{p} \cos n k\right|_{0} ^{2 \pi}=\sum_{m=1}^{p-1} \sum_{t=0}^{m} \sum_{r=0}^{t+1}\left(\begin{array}{c}
t+1 \\
r
\end{array}\right) \frac{S_{m}^{p}(m)_{1}^{(t)}(2 \pi)_{\ell}^{(m-t)} \cos n(r-1) \ell}{(-1)^{r-1} N \ell^{m-t-p}(2(\cos n \ell-1))^{t+1}}
$$

and

$$
b_{n}=\left.\frac{\ell}{\pi} \Delta_{\ell}^{-1} k^{p} \sin n k\right|_{0} ^{2 \pi}=\sum_{m=1}^{p-1} \sum_{t=0}^{m} \sum_{r=0}^{t+1}\left(\begin{array}{c}
t+1 \\
r
\end{array}\right) \frac{S_{m}^{p}(m)_{1}^{(t)}(2 \pi)_{\ell}^{(m-t)} \sin n(r-1) \ell}{(-1)^{r-1} N \ell^{m-t-p}(2(\cos n \ell-1))^{t+1}} .
$$

Proof The proof follows by applying the limits 0 to $2 \pi$ in (30) and (31), and then multiplying by $\ell / \pi$. 
Corollary 3 From (20), and using (32) and (33) for $a_{n}$ and $b_{n}$ respectively, we get the finite Fourier series for the polynomial $k^{p}$ as

$$
k^{p}=\frac{a_{0}}{2}+\sum_{n=1}^{N-1}\left(a_{n} \cos n k+b_{n} \sin n k\right)+\frac{a_{N}}{2} \cos N k, k \in\{r \ell\}_{r=0}^{2 N-1} .
$$

Corollary 4 Let $I=[0,2 \pi], \ell=\frac{\pi}{N}$, and $c>0$ be a constant. Then, for $k \in\{r \ell\}_{r=0}^{2 N-1}$, the finite Fourier series of the geometric function $c^{k}$ is given by

$$
\begin{aligned}
c^{k}=\frac{\ell}{2 \pi}\left(\frac{c^{2 \pi}-1}{c^{\ell}-1}\right)+\frac{\ell}{\pi} \sum_{n=1}^{N-1}\left(\cos n k \sum_{r=1}^{[2 \pi / \ell]} c^{(2 \pi-r \ell)} \cos n r \ell\right. \\
\left.-\sin n k \sum_{r=1}^{[2 \pi / \ell]} c^{(2 \pi-r \ell)} \sin n r \ell\right)+\frac{\ell}{2 \pi} \cos N k \sum_{r=1}^{[2 \pi / \ell]} c^{(2 \pi-r \ell)} \cos N r \ell .
\end{aligned}
$$

Proof The proof follows by taking $u(k)=c^{k}$ in (20), and then applying (15).

The following example is a verification of Corollary 4

Example 3 Taking $c=8, N=100$ and $\ell=\frac{\pi}{100}$ in (35), we have

$$
\begin{aligned}
8^{k}= & \frac{8^{2 \pi}-1}{200\left(8^{\pi / 100}-1\right)}+\frac{1}{100} \sum_{n=1}^{99}\left(\sum_{r=1}^{200} 8^{(2 \pi-r(\pi / 100))} \cos \frac{n r \pi}{100} \cos n k\right. \\
& \left.\quad-\sum_{r=1}^{200} 8^{(2 \pi-r(\pi / 100))} \sin \frac{n r \pi}{100} \sin n k\right)+\frac{1}{200} \sum_{r=1}^{200} \cos r \pi \cos 100 k, k \in\left\{\frac{r \pi}{100}\right\}_{r=0}^{199} .
\end{aligned}
$$

Theorem 3.4 Let $I=[2 \pi, 4 \pi], m \in(-\infty, \infty), \ell=\frac{\pi}{N}$. Then the finite Fourier series of rational function $\frac{1}{k^{m}}$ is given by

$$
\begin{aligned}
\frac{1}{k^{m}}= & \frac{\ell}{2 \pi} \sum_{r=1}^{[2 \pi / \ell]} \frac{1}{(4 \pi-r \ell)^{m}}+\frac{\ell}{\pi} \sum_{n=1}^{N-1}\left(\sum_{r=1}^{[2 \pi / \ell]} \frac{\cos n r \ell}{(4 \pi-r \ell)^{m}} \cos n k\right. \\
& \left.-\sum_{r=1}^{[2 \pi / \ell]} \frac{\sin n r \ell}{(4 \pi-r \ell)^{m}} \sin n k\right)+\frac{\ell}{2 \pi} \sum_{r=1}^{[2 \pi / \ell]} \frac{\cos N r \ell}{(4 \pi-r \ell)^{m}} \cos N k, k \in\{2 \pi+r \ell\}_{r=0}^{2 N-1} .
\end{aligned}
$$

Proof Taking $a=2 \pi, u(k)=\frac{1}{k^{m}}$ in (20) and applying (15), we get (36). 
The following example is a verification of the Corollary 3.4.

Example 4 Taking $m=5, N=24, \ell=\frac{\pi}{24}$ in (36), then we have $\frac{1}{k^{5}}=\frac{1}{48} \sum_{r=1}^{48} \frac{1}{(4 \pi-r(\pi / 24))^{5}}+\frac{1}{24} \sum_{n=1}^{23}\left(\sum_{r=1}^{48} \frac{\cos n r(\pi / 24)}{(4 \pi-r(\pi / 24))^{5}} \cos n k\right.$

$\left.-\sum_{r=1}^{48} \frac{\sin n r(\pi / 24)}{(4 \pi-r(\pi / 24))^{5}} \sin n k\right)+\frac{1}{48} \sum_{r=1}^{48} \frac{\cos r \pi}{(4 \pi-r(\pi / 24))^{5}} \cos 24 k, k \in\left\{2 \pi+\frac{r \pi}{24}\right\}_{r=0}^{47}$

Theorem 3.5 Let $I=[\pi / 8,17 \pi / 8], \ell=\frac{\pi}{N}$. Then, the finite Fourier series of the logarithmic function $\log k$ is given by

$$
\begin{aligned}
\log k=\frac{\ell}{2 \pi} \sum_{r=1}^{[2 \pi / \ell]} \log \left(\frac{17 \pi}{8}-r \ell\right) & +\frac{\ell}{\pi} \sum_{n=1}^{N-1}\left(\sum_{r=1}^{[2 \pi / \ell]} \log \left(\frac{17 \pi}{8}-r \ell\right) \cos n\left(\frac{17 \pi}{8}-r \ell\right) \cos n k\right. \\
& \left.+\sum_{r=1}^{[2 \pi / \ell]} \log \left(\frac{17 \pi}{8}-r \ell\right) \sin n\left(\frac{17 \pi}{8}-r \ell\right) \sin n k\right) \\
+ & \frac{\ell}{2 \pi} \sum_{r=1}^{[2 \pi / \ell]} \log \left(\frac{17 \pi}{8}-r \ell\right) \cos N\left(\frac{17 \pi}{8}-r \ell\right) \cos N k, k \in\left\{\frac{\pi}{8}+r \ell\right\}_{r=0}^{2 N-1}
\end{aligned}
$$

Proof The proof follows by taking $a=\frac{\pi}{8}, u(k)=\log k$ in (20) and applying (15).

The following example is a verification of the Corollary 3.5

Example 5 Taking $N=8, \ell=\frac{\pi}{8}$ in (37), for $k \in\left\{\frac{\pi+r \pi}{8}\right\}_{r=0}^{15}$, we have $\log k=\frac{1}{16} \sum_{r=1}^{16} \log \left(\frac{17 \pi-r \pi}{8}\right)+$ $\frac{1}{8} \sum_{n=1}^{7}\left(\sum_{r=1}^{16} \log \left(\frac{17 \pi-r \pi}{8}\right) \cos n\left(\frac{17 \pi-r \pi}{8}\right) \cos n k\right.$

$\left.+\sum_{r=1}^{16} \log \left(\frac{17 \pi-r \pi}{8}\right) \sin n\left(\frac{17 \pi-r \pi}{8}\right) \sin n k\right)+\frac{1}{16} \sum_{r=1}^{16} \log \left(\frac{17 \pi-r \pi}{8}\right) \cos 8\left(\frac{17 \pi-r \pi}{8}\right) \cos 8 k$. Taking $p=$ 2, $N=11$ and $\ell=\frac{\pi}{11}$ in (34), we have

$$
\begin{gathered}
k^{2}=\frac{301 \pi^{2}}{242}+\sum_{n=1}^{10}\left(\frac{-2 \pi^{2}}{121}\left(11-\frac{1}{1-\cos n(\pi / 11)}\right) \cos n k+\frac{22 \pi^{2} \sin n(21 \pi / 11)}{121(1-\cos n(\pi / 11))} \sin n k\right) \\
-\frac{21 \pi^{2}}{242} \cos 11 k, k \in\left\{\frac{r \pi}{11}\right\}_{r=0}^{21} .
\end{gathered}
$$

Here we provide MATLAB coding for verification FS: syms $n$ $p i .^{\wedge} 2=\left(\left(301 * p i .^{\wedge} 2\right) . / 242\right)+\operatorname{symsum}\left(\left(\left(-2 * p i .^{\wedge} 2 . / 121\right) *(11-1 . /(1-\cos (n * p i . / 11)))\right) * \cos (n *\right.$ $\left.p i)+\left(\left(22 * p i .^{\wedge} 2 . / 121\right) *(\sin (n * 21 * p i . / 11) \cdot /(1-\cos (n * p i . / 11)))\right) * \sin (n * p i), n, 1,10\right)-(2 *$ pi. $\left.{ }^{\wedge} 2 . / 242\right) *(11-1 . /(1-\cos (p i))) * \cos (11 * p i)$ 

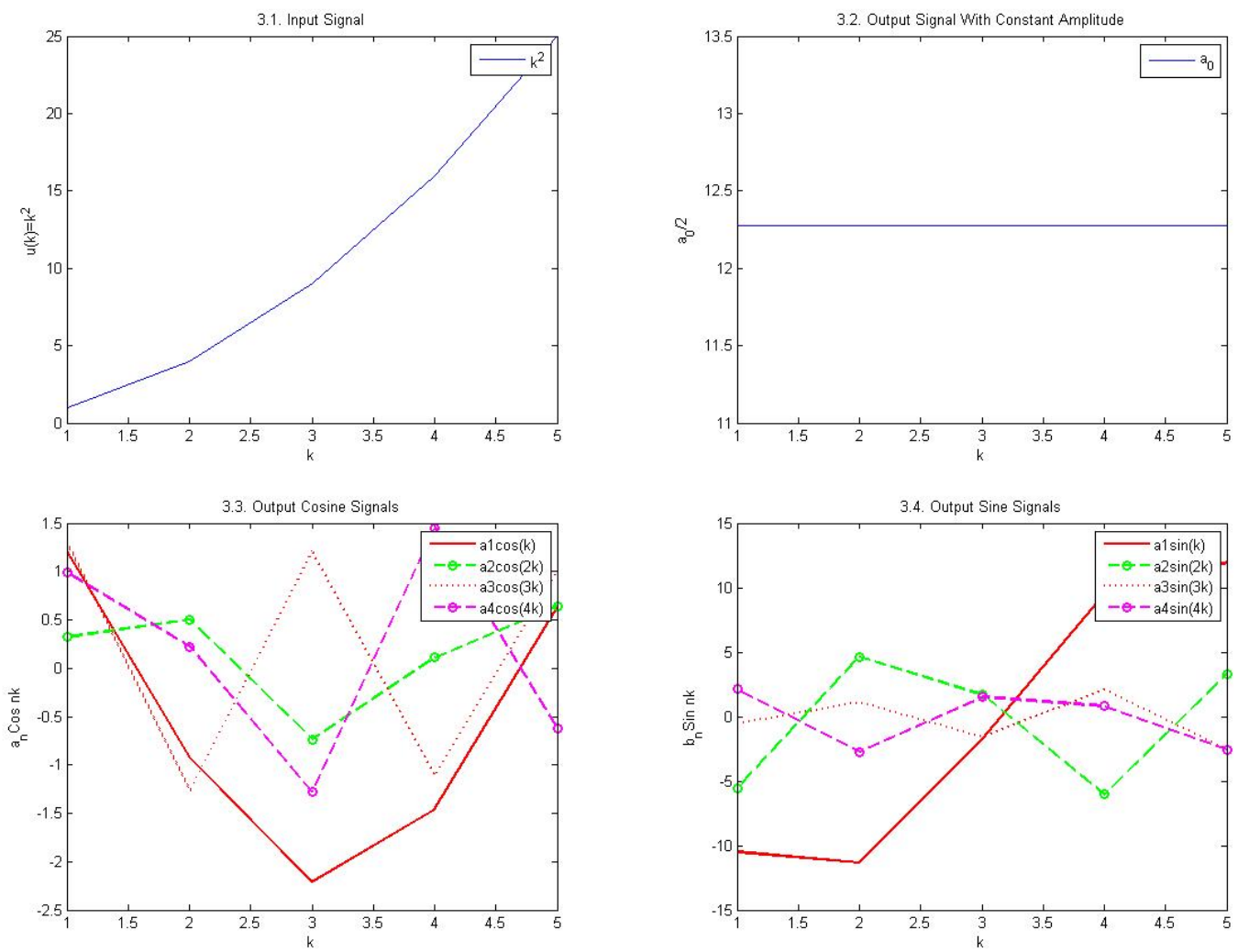

Discussion: The diagrams 3.2 to 3.4 give 9 components of the decomposition of the function $u(k)=k^{2}$ (input signal). One can get the remaining 13 components easily.

\section{Conclusion:}

The Fourier series and its transforms have wide range of applications specially in the field of digital signal process. For functions which have no usual Fourier series expression, we are able to find finite Fourier series expression (decomposition) using summation solution of generalized difference equation. The method discussed in this paper leads to several applications in signal process.

\section{References}

[1] R.P. Agarwal, Difference Equations and Inequalities, Marcel Dekker, New York, 2000.

[2] Albert Leon Whiteman, Finite Fourier Series and Cycltomy, National Academy of Sciences, Vol. 37, No. 6 (Jun. 15, 1951), pp. 373-378. 
[3] R.D.Blevins, Probability Density of Finite Fourier Series with Random Phases, Journal of Sound and Vibration (1997) 208(4), 617-652.

[4] V. Britanak and K. R. Rao, " The Fast Generalized discrete Fourier Transforms: A unified Approach to the Discrete Sinusoidal Transforms Computation," Siganal Processing, vol. 79, pp. 135-150, Dec 1999.c

[5] G.Britto Antony XAVIER, S.SAThya, S.U.VASAnthaKumar, $m$-Series of the Generalized Difference Equation to Circular Functions, International Journal of Mathematical Archive4(7), (2013), 200-209.

[6] Dennis J.Whitford And MARIO E.C. VIEIRA, Teaching time series analysis.I.Finite Fourier analysis of ocean waves, American Journal of Physis.69(4), April 2001.

[7] J.Y. LAI AND J.R. BoOKER, Application of Discrete Fourier Series to the Finite Element Stress Analysis of Axi-Symmetric Solids, International Journal for Numerical Methods in Engineering, Vol.31,619-547, 1991.

[8] M.Maria Susai Manuel, G.Britto Antony Xavier and E.Thandapani, Theory of Generalized Difference Operator and Its Applications, Far East Journal of Mathematical Sciences, 20(2) (2006), 163 - 171.

[9] M.Maria Susai Manuel, G.Britto Antony Xavier, D.S.Dilip and G.DominiC BABU, Nonexistence of Solutions of Certain Type of Second Order Generalized $\alpha$-Difference Equation in $\ell_{2}(\alpha(\ell))$ and $c_{0(\alpha(\ell))}$ Spaces, Southeast Asian Bulletin of Mathematics (2016) 40: 329340.

[10] I.J.Schoenberg, The Finite Fourier Series and Elementary Geometry, Mathematical Association of America, Vol. 57, No. 6 (Jun. - Jul., 1950), pp. 390-404.

[11] Steven W. Smith, The Scientist and Engineer's Guide to Digital Signal Processing, Second Edition, California Technical Publishing San Diego, California, 1999. 